\title{
BICEP2 and the Gravitino Mass: The Questionable Result
}

\author{
AC Tahan ${ }^{1}$ \\ ${ }^{1}$ ETHERMED $_{\text {тм }}$, Cambridge, MA, USA \\ Correspondence: AC Tahan, ETHERMED, PO Box 391987, Cambridge, MA 02139, USA. E-mail: \\ actahan@ethermed.com
}

Received: April 30, $2014 \quad$ Accepted: June 23, $2014 \quad$ Online Published: August 8, 2014
doi:10.5539/mas.v8n5p30
URL: http://dx.doi.org/10.5539/mas.v8n5p30

\begin{abstract}
The gravitino has been explained to exist with a low mass (Tahan 2013). BICEP2 results support a mass close to $10^{14} \mathrm{GeV}$. Though the BICEP2 conclusions have been celebrated by numerous physicists with fewer scientists indicating problems with the data, $r=0.20_{-0.05}^{+0.07}$ from BICEP2 (BICEP2 Collaboration 2014) can be understood improbable by simply focusing on the mass of the gravitino. This manuscript presents a method involving increasing string vibrations to measure the gravitino mass in the laboratory.
\end{abstract}

Keywords: gravitino, BICEP2, dark matter, string experimentation

\section{Background}

In attempts to understand how gravity fits in the Standard Model the gravitino has been an unavoidable subject since it is a superpartner of the graviton, specifically regarding supergravity analogous to electroweak theory $\mathrm{W}$ and $\mathrm{Z}$ bosons. Accordingly, the gravitino has been well-studied including for troubling Universe topics as dark matter, which has forced the issue of the superparticle mass if wishing to study gravity in the evolution of the Universe. Inflation emerged as the most popular scenario for the development of the Universe and consequently the gravitino has been tied to different inflation theories. The mass of the superpartner has usually been a primary point in the ideas related to how the gravitino can exist with inflation, even having led to disfavoring gravitino dark matter because of discussions regarding how the mass could have prevented the existence of the Universe, e.g. the gravitino problem (Weinberg, 1982). Therefore, the gravitino became a less popular topic of study, particularly considering that evidence for supersymmetry or string theory has been nonexistent.

A recent report from the BICEP2 collaboration has reintroduced the possibility for the existence of the gravitino with a large mass, which has not been a primary topic in papers referencing the findings. The mass may not have been well-discussed so far since perhaps it has been considered, like other particles, an unavoidable result of the overall events suggested by the BICEP2 data. Still, the BICEP2 work discussing quantum gravity requires clarification for the existence of a high mass gravitino, particularly considering past studies. By considering the mass in relation to work performed with an instrument that permitted graviton appearance in the lab (Tahan 2011) while remembering theoretical studies of the gravitino, the high mass alone would suggest that the BICEP2 findings are questionable.

\subsection{Introduction}

Though the BICEP2 team has not presented all data and the $\mathrm{r}=0.20_{-0.05}^{+0.07}$ result is inconsistent with Wilkinson Microwave Anisotropy Probe (WMAP) satellite (Hinshaw et al., 2013) and Planck satellite information (Planck Collaboration, 2014) thus far, the BICEP2 report has been received predominantly with great fanfare. The BICEP2 conclusions support quantum gravity, which again would suggest the existence of the gravitino. However, according to the BICEP2 findings the gravitino mass would have to be about $10^{14} \mathrm{GeV}$, which was less acceptable before the BICEP2 report due to reasons including a large mass and decay products possibly influencing overclosure of the Universe (Jeong \& Takahashi, 2012) or Big Bang Nucleosynthesis (BBN) (Kawasaki, Kohri, Moroi, \& Yotsuyanagi, 2008).

Apart from scholars using the BICEP2 results to support their work, the majority of subsequent papers have discussed how the visible Universe could exist involving inflation in consideration of the data, though researchers have written that $B$-mode polarization is not necessarily evidence for inflation (Brandenberger, 2011). Few publications have addressed the limitations of the BICEP2 instrumentation, e.g. compared to the Planck satellite regarding operating frequencies. Rarely have scientists scrutinized the data, including specific points on 
graphs that may not fit explanations. Little criticism has been presented regarding unusual methods the BICEP2 team may have used to analyze or compare data particularly to information from other projects, and minimal discussion has existed regarding what the BICEP2 team may have overlooked, e.g. the Wolleben New Loop (Wolleben, 2007; Liu, Mertsch, \& Sarkar, 2014). Papers indicating that BICEP2 data diverge from theory, for instance not allowing string theory and inflation to coexist (Sakellariadou 2014), have not expressed the results to be worrisome but instead have explained the discrepancies to mean that BICEP2 has shown that events in the progression of the Universe need to be modified to allow for BICEP2 findings. And the gravitino seldom has been mentioned; manuscripts suggesting that the high mass makes the BICEP2 results questionable are uncommon. Instead scientists have proposed situations to allow for a large gravitino mass (Fan, Jain, \& Ozsoy, 2014)

The inability of synchrotron teams to find supersymmetry suggests a broken symmetry, permitting more massive superparticles than related Standard Model bodies. Prior to the BICEP2 conclusions certain symmetry breaking theories permitting near apart from significantly above $1 \mathrm{TeV}$ gravitinos were disfavored (Jeong, Park, Stewart, \& Kadota, 2004, Endo, Hamaguchi, \& Takahashi, 2006), additional references could have been presented. Manuscripts have not properly clarified what type of supersymmetry breaking would have allowed for events including the massive gravitino, i.e. particular scenarios conflict discounted proposals or ideas in different papers are contradictory or incompatible.

\section{Tool for Explorations beyond the Standard Model}

A tool for explorations beyond the Standard Model exists that has allowed for the visualization of an open string and D-brane (Tahan, 2011). Results supported a sub-keV gravitino mass, being the lightest superparticle (LSP) involving minor violation of R-parity and potentially dark matter that includes fifth dimension primordial black holes (Tahan, 2013). With inclusion of a slight violation of R-parity the gravitino LSP has a duration beyond the lifetime of the Universe (Takayama \& Yamaguchi, 2000) and primordial black holes and the small massed gravitinos would be cold dark matter in consideration of entropy (Lemoine, Moultaka, \& Jedamzik, 2007). Additionally, work with the Figure 1 set-up that showed the prevalence of branes supported the existence for a division of an underlying superparticle and graviton area and the Standard Model visible sector.

Conclusions due to use of the Figure 1 set-up force the BICEP2 results in consideration of the gravitino mass to be considered highly improbable and suggest that a method for determining the validity of the BICEP 2 work would be to use the Figure 1 technique to understand the mass of the gravitino. The symmetry breaking due to the set-up was periodic. By that, multiple Hydrogen branes were influenced during approximately hour long trials so that by surrounding the set-up with proper detectors, several opportunities for gravitino mass measurements would arise per trial.

The gravitino weakly couples to matter thereby making direct detection doubtful at colliders. Researchers preferring late decay in the Cosmos especially involving the LSP gravitino have proposed discovery through next to lightest superparticle (NLSP) decay. NLSP decay to gravitinos would be unlikely if the gravitino is the LSP with slight violation of R-parity. The NLSP would be ephemeral and decay straight to R-parity even particles (Takayama \& Yamaguchi, 2000), lowering the possibility for gravitinos to be found at synchrotrons thereby suggesting that solely the Figure 1 procedure could permit gravitino detections including perhaps other superpartners if incorporating the right detectors with the set-up. Also, this work should not be considered to disfavor inflation. Describing small massed gravitinos as the LSP and cold dark matter support a relationship between supersymmetry and inflation (Tahan, 2013). This manuscript is intended only to suggest that disagreement regarding the accuracy of BICEP2 findings, particularly if upcoming data as from the Planck satellite do not resolve questions related to the results, possibly can be settled with a direct measure of the gravitino mass using the Figure 1 set-up. Though regardless of future collaboration reports, only the Figure 1 instrument allows for specific studies and appearances, e.g. the direct observations of strings (Tahan, 2011). 


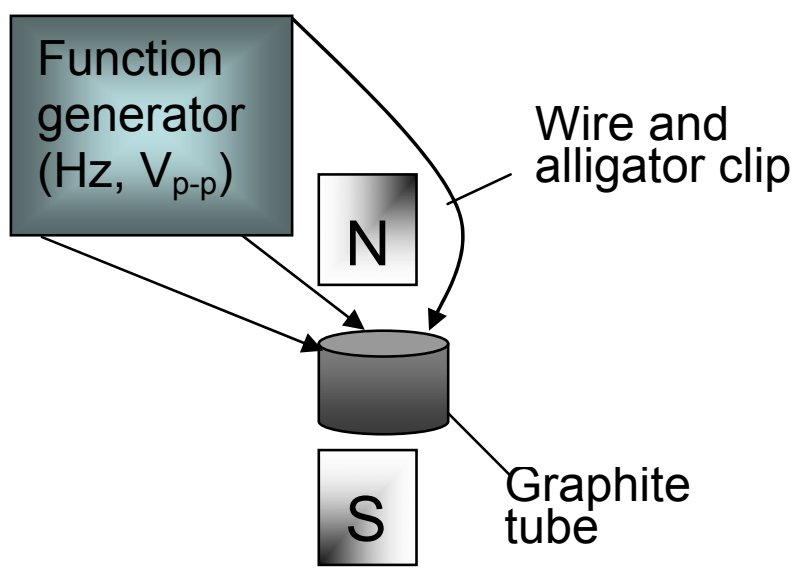

Figure 1. Basic apparatus

Laser light (Quartet Standard Laser Pointer), $\approx .017 \mathrm{~m}$ from the middle and about $.01-.02 \mathrm{~m}$ from the bottom of a graphite tube creating an approximately $.004-.005 \mathrm{~m}$ line on the side of the tube closest to the $\mathrm{S}$ pole of a gap magnet, for the D-brane trials was removed for Figure 1, which is the basic set-up. Incorporation of the laser was to examine the possibility of the bending of spacetime resulting from gravitons emerging due to symmetry breaking (Tahan, 2012) and interacting with the tube, imparting mass-energy thereby causing the tube to bend spacetime sufficiently to alter the direction of the light. Sulfuric acid $\left(\mathrm{H}_{2} \mathrm{SO}_{4}\right)$ (typically $20 . \mathrm{mL}, 96 \%$ concd. Mallinckrodt Analytical Reagent, ACS) was in the tube for each experiment. Approximately $99.7 \mathrm{kPa}$ and $98-100 \%$ were the barometric pressure and relative humidity (Boston, MA, CW1378) during the largest laser light D-brane trial (Tahan, 2011). The tube was clamped in the static magnetic field, $\mathrm{N}$ and $\mathrm{S}$ being the poles of the gap magnet, measuring about 2000 Gs. Small distance variations toward the S pole were immaterial: $\approx .004-.012 \mathrm{~m}$ should allow for observations. Wires from a breadboard to which the function generator was attached to a LED supplied $\approx 2 \mathrm{~Hz}(2.000-2.012 \mathrm{~Hz})$ and $\mathrm{V}_{\mathrm{p}-\mathrm{p}}=4.312-4.437$, predominately 4.375 , to the graphite container. The low frequency was not intended to alter Hydrogen alignment in the magnetic field. The set-up was ineffective when the magnet was removed. One wire with an alligator clip is required; three wires were connected triangularly to the top of the tube to be sure that the frequency and amplitude were being supplied--in view of potential inoperability in an experiment as because of clip corrosion from acid exposure. The graphite tube, clamp that held the tube, and the stand to which the clamp was attached that included the $\approx .153 \mathrm{~kg}$ clamp holder were $\approx .098-.099 \mathrm{~kg}$, near $.230 \mathrm{~kg}$, and about $1.458 \mathrm{~kg}$ respectively. The set-up requires the frequency and amplitude, tube (Crucible, Saed/Manfredi G40, 1.5 "OD x 1.25 "ID x $3.75 " \mathrm{DP})$, constant magnetic field strength ( $\approx 2000 \mathrm{Gs})$, and concentrated sulfuric acid--the only components always needed. Manners for supplying the parts can be tested, for example Hydrogen from a different source than $\mathrm{H}_{2} \mathrm{SO}_{4}$. But modifications to the components may be needed depending on the change: e.g. the amplitude may require adjustment. When utilizing only one wire, if the set-up is inoperable and the clip is well-attached and not requiring replacement, especially if a researcher had attached the three wires dissimilarly modification of the amplitude may be necessary, imagining the three wires to have supplied quanta differently for suitable string vibrations. The graphite tube and acid supply should be changed occasionally to avoid variables, for instance impurities in the acid or eventual deformation of the tube due to the acid that may inhibit regular frequency conduction. The acid should be replaced in the tube for each trial unless the examiner wishes to test a particular aspect of the set-up involving the acid. The Figure 1 apparatus permits periodic appearances of superpartners and gravitons that can be presented similarly to a reaction: gluon, gluon, gluon, gluino, gluino, gluino, photon, proton, electron, $\mathrm{W}^{-}$boson, Higgs boson, $\mathrm{Z}$ boson, axion, dilaton $\rightarrow$ graviton, gravitino, neutron, electron neutrino, graviscalar, graviphoton, photon, photon, photon, sneutrino, selectron, saxion (Tahan, 2012).

Use of the set-up allowed for the writing of the reaction in the Figure 1 description that was utilized for a calculation $125.81 \mathrm{GeV}$ of the Higgs Boson mass (Tahan, 2012). After learning that the calculated mass was not dissimilar from collider discoveries (Incandela, 2012; Gianotti, 2012), in view of the reaction the gravitino was 
thought should not be greater than $1 \mathrm{keV}$, also while considering theoretical work instructing that gravitinos with a larger mass would have overclosed the Universe (Cho \& Uehara, 2004)--appreciating the superparticle as the LSP therefore avoiding issues with BBN. The gravitino being a light superpartner, particularly the LSP, would support the body as dark matter (Giudice \& Rattazzi, 1999; Takayama \& Yamaguchi, 2000). The reaction can be utilized to predict bodies and rewritten to contain or remove particles if understood required primarily after using suitable detectors with the set-up.

\section{String Experimentation}

Prior to work with the Figure 1 set-up, strings were unknown to exist and scientists believed that the predicted, required high energies to observe strings made the possibility of related work unlikely. The BICEP2 report being supportive of quantum gravity, i.e. the graviton, would be suggestive of the existence of strings since quantum gravity is well-accepted among theorists as a string theory (Scherk \& Schwarz, Phys. Letters, 1974). The Figure 1 method is the only tool that permits the direct visualization of strings, including allowing for the guiding of gravitons to locations that results in the gravitons imparting mass-energy that occurred in the lab for the Figure 1 graphite tube, causing the curving of spacetime and resultantly the bending of the laser light around the tube (Tahan, 2011).

For experiments, the initial hypothesis for use with the Figure 1 apparatus that led to gravitons in the laboratory concerned principles of string theory regarding Hydrogen. The method involved $2 \mathrm{~Hz}$ with a particular amplitude to create vibrations of the Hydrogen brane that resulted in increasing energy and eventually symmetry breaking that was periodic in the concentrated $\mathrm{H}_{2} \mathrm{SO}_{4}$ supply for Hydrogen, i.e. symmetry breaking for various Hydrogen happening at distinct times in the usual roughly one hour experiments. Specifically, the foundation of Hydrogen (protons) is the string, principally gluon field strings, with tension that can be altered with a magnetic field thereby permitting increased vibrations due to electromagnetic energy of the proper frequency and amplitude in consideration of the manipulated tension.

The escalating vibrations of a string are identical to an increasing energy (Scherk \& Schwarz, Nucl. Phys. B, 1974). The $2 \mathrm{~Hz}$ was useful mainly due to influencing gluon field strings: a strong force string underlying a uniform field of quanta (Yang \& Mills, 1954). The field strength (coupling constant) between quarks is consistent (Mills, 1994); as energy increased the coupling constant decreased (Aharony, Gubser, Maldacena, Ooguri, \& Oz, 2000). Hydrogen maintaining alignment in the magnetic field caused the tension of the Hydrogen brane and associated strings to stay continuous thus allowing for growing vibrations. Consequently ultimately the proton, involving electron capture (Tahan, 2012), fell apart thereby permitting the glueball and symmetry breaking. In other words, if appreciating Yang-Mills theory to be a quantum gauge theory with the gauge group $\mathrm{SU}(\mathrm{N})$ being equivalent to a string theory with $1 / \mathrm{N}$ as the string coupling constant ('t Hooft, 1974, Maldacena 1998) the strong force coupling constant steadily reached the limit $1 / 3$, a large $\mathrm{N}$ analogous to a vibration limit (Aharony, Gubser, Maldacena, Ooguri, \& Oz, 2000), because of the incessant $2 \mathrm{~Hz}$ quanta that resulted in deconfinement. Namely, the coupling constant can be considered inversely proportional to an energy that corresponds to the gluon field. Increasing string vibrations result in a gluon field advancing toward a limit infinity ('t Hooft, 1974). At high energy due to approaching the limit, the coupling constant became zero that permitted liberation of gluon strings. The glueball occurring from the coalescing of the strings before the massive superpartners of the graviton (Tahan, 2012) suggested that confinement and the Higgs mechanism are attributes of the strong force string (Fradkin \& Shenker, 1979).

Using $2 \mathrm{~Hz}$ with a particular amplitude could allow the Figure 1 method to be thought a low energy technique. However, what should be remembered is that a string is energy. Specifically, a problem for scholars has been to define energy, which is resolved by equating energy to a string that becomes a greater quantity of energy the more it vibrates. The $2 \mathrm{~Hz}$ was a resonant frequency for the altered strings of the Hydrogen in the magnetic field, creating steadily growing string vibrations thereby increasing energy that influenced the structure of the Hydrogen. The required point at which the symmetry breaking process occurred was reached as the strings vibrated that can be visualized with the involved particles of the Figure 1 reaction, which again can be edited with future studies using the instrument. By that, the needed high energy for deconfinement was provided by the Hydrogen, videlicet its strings.

The ease of set-up permits testing predictions inexpensively and in a typical laboratory setting. Thus, possible due to the Figure 1 apparatus string experimentation is the practical use of string resonance. Through the manipulation of strings the Figure 1 method can lead to globally needed applications including using the tool for research, for example to manufacture gravitons and superpartners as the gravitino. 


\section{Conclusions}

This manuscript emerged due to concern regarding how quickly the BICEP2 report was applauded and accepted in the scientific community (Cowen 2014), though again a few scientists--particularly experimentalists--have expressed displeasure regarding BICEP2 data. The acknowledgment is not immensely surprising if considering Large Hadron Collider (LHC) results.

Physicists are bothered that LHC teams have not presented more discoveries especially beyond the Standard Model (Grant, 2013), to the extent of a panic that would explain swift approval of the BICEP2 information that was unforeseen. Specifically, a theorist makes predictions consistent with findings from experiments. Unexpected data provide more opportunities for explanations. And though the Higgs boson result has been celebrated disappointment seems to be the main product for the first years of the LHC, from which the conclusions were anticipated.

With the emergence of string experimentation due to the Figure 1 technique frustration or worries regarding the future of theoretical physics or physics studies generally should not exist. The method is a low-cost, facile set-up and permits theoretical work and experiments on a lab bench (Tahan, 2012). Collider teams not having detected supersymmetry should require the instrument to be accepted at least alternatively for string and superpartner examinations, as to measure the gravitino mass, since it is the only tool that manifests superparticles apart from strings: for instance the open string and D-brane of the laser light when part of the Figure 1 set-up (Tahan, 2011). For improved opportunities to observe D-branes with open strings pristine graphite tubes should be used since more graphite will leak to the acid, where gravitons emerge and will interact with the atoms thereby creating larger high gravitational areas that will greater influence the light (Tahan, 2011; Tahan, 2013). Therefore, instead hope should subsist if the apparatus is appreciated as the viable tool for studies outside the Standard Model.

\section{References}

Aharony, O., Gubser, S. S., Maldacena, J., Ooguri, H., \& Oz, Y. (2000). Large N field theories, string theory and gravity. Phys. Rep., 323, 183-386. http://dx.doi.org/10.1016/S0370-1573(99)00083-6

BICEP2 Collaboration, P. A. R. Ade et al. (2014). BICEP2 I: Detection of B-mode polarization at degree angular scales, arXiv:1403.3985 [astro-ph.CO].

Brandenberger, R. H. (2011). Is the spectrum of gravitational waves the "Holy Grail" of inflation? arXiv:1104.3581v1 [astro-ph.CO].

Cho, G. C., \& Uehara, Y. (2004). Cosmological gravitino problem confronts electroweak physics. Phys. Rev. 69D, 075003. http://dx.doi.org/10.1103/PhysRevD.69.075003

Cowen, R. (2014). Telescope captures view of gravitational waves. Nature, 507, 281-283. http://dx.doi.org/10.1038/507281a

Endo, M., Hamaguchi, K., \& Takahashi, F. (2006). Moduli-induced gravitino problem. Phys. Rev. Lett. 96, 211301. http://dx.doi.org/10.1103/PhysRevLett.96.211301

Fan, J. J., Jain, B., \& Ozsoy, O. (2014). Heavy gravitino and split SUSY in the light of BICEP2, arXiv: $1404.1914 \mathrm{v} 1$.

Fradkin, E., \& Shenker, S. H. (1979). Phase diagrams of lattice gauge theories with Higgs fields. Phys. Rev., 19D, 3682-3697. http://dx.doi.org/10.1103/PhysRevD.19.3682

Gianotti, F. (2012). Update on the Standard Model Higgs searches in ATLAS, CERN Seminar.

Giudice, G. F., \& Rattazzi, R. (1999). Theories with gauge-mediated supersymmetry breaking. Phys. Rept., 322, 419-499. http://dx.doi.org/10.1016/S0370-1573(99)00042-3

Grant, A. (2013). Hard times for theorists in a post-Higgs world. Science News, 183(13), 22-25. http://dx.doi.org/10.1002/scin.5591831323

Hinshaw, G. et al. (2013). Nine-year Wilkinson Microwave Anisotropy Probe (WMAP) observations: Cosmological parameter results, arXiv:1212.5226v3 [astro-ph.CO].

Incandela, J. (2012). Update on the Standard Model Higgs searches in CMS, CERN Seminar.

Jeong, D., Park, W. I., Stewart, E. D., \& Kadota, K. (2004). Modular cosmology, thermal inflation, baryogenesis and a prediction for particle accelerators. JHEP, 11, 046. http://dx.doi.org/10.1088/1126-6708/2004/11/046

Jeong, K. S., \& Takahashi, F. (2012). A Gravitino-rich Universe, TU-921, arXiv:1210.4077v1 [hep-ph].

Kawasaki, M., Kohri, K., Moroi, T., \& Yotsuyanagi, A. (2008). Big-Bang nucleosynthesis and gravitino. Phys. 
Rev., 78D, 065011. http://dx.doi.org/10.1103/PhysRevD.78.065011

Lemoine, M., Moultaka, G., \& Jedamzik, K. (2007). Natural gravitino dark matter in SO(10) gauge mediated supersymmetry breaking. Phys. Lett., 645B, 222-227. http://dx.doi.org/10.1016/j.physletb.2006.12.025

Liu, H., Mertsch, P., \& Sarkar, S. (2014). Fingerprints of Galactic Loop I on the Cosmic Microwave Background, arXiv:1404.1899v1 [astro-ph.CO].

Maldacena, J. (1998). The large N limit of superconformal field theories and supergravity. Adv. Theor. Math. Phys., 2, 231-252.

Mills, R. L. (1994). Space, time, and quanta: An introduction to contemporary physics, Part III. New York: W.H. Freeman.

Planck Collaboration, P. A. R. Ade et al. (2014). Planck 2013 results. XVI. Cosmological parameters, arXiv:1303.5076v3 [astro-ph.CO].

Sakellariadou, M. (2014). How well do we understand the thermal history of the Universe? Implications of the recent BICEP2 data, KCL-PH-TH/2014-9, arXiv: 1403.6688v1 [astro-ph.CO].

Scherk, J., \& Schwarz, J. H. (1974). Dual models and the geometry of space-time. Phys. Letters., 52B, 347-350. http://dx.doi.org/10.1016/0370-2693(74)90059-8

Scherk, J., \& Schwarz, J. H. (1974). Dual models for non-hadrons. Nucl. Phys., 81B, 118-144. http://dx.doi.org/10.1016/0550-3213(74)90010-8

't Hooft, G. (1974). A planar diagram theory for strong interactions, Nucl. Phys., 72B, 461-473. http://dx.doi.org/10.1016/0550-3213(74)90154-0

Tahan, A. C. (2011). Exposing strings in the laboratory with a novel technique. Appl. Phys. Res. 3(2), 39-51. http://dx.doi.org/10.5539/apr.v3n2p39

Tahan, A. C. (2012). Diagrammatic presentation for the production of gravitons and supersymmetry. Mod. Appl. Sci., 6(9), 76-83. http://dx.doi.org/10.5539/mas.v6n9p76

Tahan, A. C. (2013). Low mass gravitino: Re-introducing the superpartner as dark matter with consideration to inflation due to experimentation. Mod. Appl. Sci., 7(12), 43-55. http://dx.doi.org/10.5539/mas.v7n12p43

Takayama, F., \& Yamaguchi, M. (2000). Gravitino dark matter without r-parity. Phys. Lett., 485B, 388-392. http://dx.doi.org/10.1016/S0370-2693(00)00726-7

Weinberg, S. (1982). Cosmological constraints on the scale of supersymmetry breaking. Phys. Rev. Lett., 48, 1303-1306. http://dx.doi.org/10.1103/PhysRevLett.48.1303

Wolleben, M. (2007). A new model for the Loop-I (The North Polar Spur) Region. Astrophys. J., 664, 349-356. http://dx.doi.org/10.1086/518711

Yang, C. N., \& Mills, R. L. (1954). Conservation of isotopic spin and isotopic gauge invariance. Phys. Rev., 96, 191-195. http://dx.doi.org/10.1103/PhysRev.96.191

\section{Copyrights}

Copyright for this article is retained by the author(s), with first publication rights granted to the journal.

This is an open-access article distributed under the terms and conditions of the Creative Commons Attribution license (http://creativecommons.org/licenses/by/3.0/). 